\title{
FLORULA DE LOS PREDIOS FORESTALES LOS PINOS Y LAS PALMAS CAYUMAPU, VALDIVIA
}

Florula of the forest farms Los Pinos and Las Palmas, Cayumapu, Valdivia

C.D.O.: 181.1

Federico SCHLEGEL y Osvaldo MARTINEZ

Instituto de Silvicultura, Facultad de Ciencias Forestales, Universidad Austral de Chile, casilla 853, Valdivia, Chile.

\section{RESUMEN}

El estudio de la flora de los predios forestales Los Pinos y Las Palmas, ubicados sobre la ribera sur del río Cayumapu, Valdivia, dio como resultado un catálogo de 213 especies vasculares, de las cuales $150(70,42 \%)$ son nativas y $63(29,58 \%)$ introducidas naturalizadas. Como nativas ocurren 30 especies arbóreas y 23 arbustivas. Además se encontraron 4 especies arbóreas y 4 arbustivas introducidas naturalizadas. El espectro biológico se compara con otros similares y se determinan las especies nativas de manifiesta escasez.

\section{SUMMARY}

The floristical study on the forest farm Los Pinos and Las Palmas, bordering the Cayumapu river near Valdivia, produced a catalogue of 213 vascular species. 150 of them $(70,42 \%)$ are natives and $63(29,58 \%)$ introduced naturaliced species. 30 native trees and 23 shurb species are present. 4 introduced and naturaliced trees and 4 shurb species were identified.

The biological spectrum is compared with others from different localities, and the extremely rare species are named.

\section{INTRODUCCION}

El presente estudio está constituido fundamentalmente por una lista de las especies nativas y naturalizadas que se desarrollan en los predios Los Pinos y Las Palmas, pertenecientes a la Universidad Austral de Chile. Ambos se sitúan sobre la ribera sur del río Cayumapu de la provincia de Valdivia y abarcan una superficie total de 742 ha.

La flora de los alrededores de Cayumapu de la provincia de Valdivia no ha sido estudiada en detalle hasta el momento. Existen antecedentes muy generales, como lo son las descripciones florísticas y vegetacionales de REICHE (1934), HAUMAN (1913), OBERDORFER (1960) y MONTALDO (1966).
El propósito de esta investigación florística detallada es crear una base para futuras investigaciones florísticas, fitosociológicas y fitogeográficas y ecológicas de los alrededores de Valdivia. Por otra parte, cumple con la finalidad de proporcionar antecedentes para el conocimiento y manejo de los recursos naturales renovables del centro-sur de Chile.

\section{MATERIAL Y METODO}

Se estudiaron las plantas vasculares herborizadas durante los años 1973 hasta 1978 en los sitios más representativos para los diferentes tipos fisionómicos de vegetación. Estos últimos pueden clasificarse en: a) Matorrales: comunidades herbáceo-arbustivas en áreas de explotación maderera reciente y arbustivo-arbóreas en zonas de alteraciones más antiguas, b) Bosques pantanosos: comunidades arbustivo-arborecentes a orillas de cursos de agua o en terrenos de drenaje limitado y c) Bosque nativo secundario: comunidades arbóreo-arbustivas inalteradas por un período prolongado.

El material recolectado se determinó en base a la bibliografía disponible en la Universidad Austral de Chile, comparándolo con ejemplares de herbario del Instituto de Botánica de la misma universidad y del Departamento de Botánica de la Universidad de Concepción. En esta última se consultaron además, las monografías existentes. Con las especies vegetales determinadas se confeccionó un catálogo en el que se ordenaron alfabéticamente familias, géneros y especies siguiendo el sistema de MUÑOZ (1966). De las especies se entrega información, sobre su forma de vida según MUELLER-DOMBOIS y ELLEMBERG (1971), nombrevernáculo según MUÑOZ( 1966), lugar y fecha de colección, abundancia, distribución en el área de estudio y distribución general.

Los ejemplares botánicos, debidamente clasifi- 
cados pasaron a formar parte del herbario del Instituto de Silvicultura de la Facultad de Ciencias Forestales de la Universidad Austral de Chile. Los duplicados fueron donados al herbario del Departamento de Botánica de la Universidad de Concepción.

Características del área del estudio. El área de estudio se encuentra ubicada geográficamente en las coordenadas $39^{\circ} 45^{\prime}$ Lat. Sur y $73^{\circ} 10^{\prime}$ Long. Oeste, sobre la ribera sur del río Cayumapu, a 15 $\mathrm{km}$. de Valdivia, bordeando la carretera al norte. $\mathrm{Su}$ geomorfología se caracteriza por terrenos intermedios ondulados a quebrados. Los cursos de agua fluyen hacia el norte al río Cayumapu.

El área de estudio se encuentra ubicada entre las altitudes de 20 y 165 m s.n.m.

El clima corresponde al tipo climático cfsb, o sea, templado lluvioso con influencia mediterránea (FUENZALIDA， 1965). Térmicamente la provincia de Valdivia está situada entre las isotermas anuales de 11 y $12^{\circ}$. Los vientos predominantes son el norte y el oeste, ocurriendo entre abril y septiembre y entre octubre y febrero respectivamente.

La precipitación media anual asciende a 2.292 $\mathrm{mm}$. Existe una alta concentración de ella en los meses invernales y suelen presentarse períodos de sequía estivales de carácter ocasional (HUBER, 1970).

Los suelos corresponden a la serie Correltué y tienen las siguientes características generales: son suelos rojo-arcilloso, derivados de ceniza volcánica antigua, que se depositan sobre el complejo metamórfico de la costa. Son profundos y de texturas moderadamente finas. Poseen una alta porosidad total y por su buena estructura permiten una adecuada aireación y drenaje (IREN, 1978).

La vegetación del área estudiada ha sido alterada por un extensivo uso agropecuario, de modo que las comunidades vegetales existentes se encuentran en la actualidad en diversas etapas sucesionales. En grandes extensiones la vegetación nativa e introducida naturalizada ha sido casi totalmente desplazada por bosques puros de Pinus radiata D. Don, Pseudotsuga menziessi (Mirb,) Franco, Cupressus lusitanica Mili., Eucalyptus globulus Labill, y Acacia melanoxylon R. Br.

Esto significa que las comunidades vegetales y secundarias han quedado relegadas a linderos de plantaciones, fondos o pendientes pronunciadas de quebradas, terrenos con drenaje limitado y también al interior de plantaciones recientes.

\section{CATALOGO DE LA FLORA DE LOS PREDIOS LOS PINOS Y LAS PALMAS CAYUMAPU, VALDIVIA}

\section{ARCHEGONIATAE}

\section{ADIANTACEAE}

Adiantum chilense Kaulf. (H caesp) 'culantrillo'.

(159 OM.) Los Pinos, sección 23, Laguna

Grande. 27-01-76. Distr. predios: en lugares húmedos y sombríos, poco frecuente. Distr. gral.: Coquimbo a Estrecho de Magallanes.

\section{ASPIDIACEAE}

Ctenitis spectabilis (Kaulf.) C.Chr. (H caesp).

Non col. Distr. predios: lugares sombríos y húmedos, poco frecuente. Distr. gral.: Coquimbo a Aysén.

Polystichum chilense (Chris.) Diels (L.) Roth (H. caesp).

Non col. Distr. predios: en lugares húmedos y sombríos, poco frecuente. Distr. gral.: Valdivia a Aysén.

\section{ASPLENIACEAE}

Asplenium dareoides Desv. ( $\mathrm{H} \mathrm{E}$ herb)

(296 OM.). Las Palmas, sección 7, plantación Sequoia 1972. 9-04-76. Distr. predios: caminos sin uso, con ambientes húmedos y de mucha sombra, epífita en árboles, abundante. Distr. gral.:
Coquimbo a Magallanes.

\section{BLECHNACEAE}

Blechnum auriculatum. Desv. (H caesp) 'Palmilla'.

(262 A1.). Las Palmas, sección 9. 7-11-73. Distr. predios: lugares húmedos y plantaciones nuevas, muy abundante. Distr. gral.: Coquimbo a Magallanes.

Blechnum blechnoides. (Bory) Keyserl. (H caesp) 'Palmilla'.

(7083 FS.). Las Palmas, sección 7, quebrada Patagüillas, abundante. 25-10-78. Distr. gral.: Curicó a Aysén.

Blechnum chilense (Kaulf.) Mett. (H caesp) 'Costilla de vaca'.

Non col. Las Palmas, sección 7, abundante. Distr. gral.: Coquimbo a $49^{\circ}$ lat. S.

Blechnum hastatum. Kaulf. (H caesp) 'Costilla de vaca'.

(7081 FS.). Las Palmas, sección7, quebrada Patagüillas, abundante. 25-10-78. Distr. predios: terrenos inundables, vertientes y bosques de pan- 
tano. Distr. gral.: Coquimbo a Magallanes.

Blechnum magellanicum. (N. Desv.) Mett. (Ch suff) 'Palmilla'.

(8231 FS.). Los Pinos, sección 36, P. radiata 1947. 16-10-87. Distr. predios: lugares sombríos y húmedos, muy escaso. Distr. gral.: desde la Cordillera de San Fernando hasta Tierra del Fuego.

\section{DENNSTAEDTIACEAE}

Hypolepis rugosula var poeppigii. (Kunze) C. Chr. (H caesp).

(7082 FS.). Las Palmas, sección 7, quebrada Patagüillas, abundante. 25-10-78. Distr. predios: en bosque nativo secundario, lugares húmedos y sombríos, poco frecuente. Distr. gral.: Valparaíso a Aysén.

\section{EQUISETACEAE}

Equisetum bogotense. H.B.K. (e $\mathrm{H}$ caesp) 'Hierba del Platero'.

(7037 FS.). Los Pinos, sección 23, Laguna Grande. 3-11-77. Distr. predios: orillas sombrías, de cursos de agua, abundante. Distr. gral.: Coquimbo a Aysén.

\section{GLEICHNIACEAE}

Gleichenia Guadripartita (Poir.) More (H herb rept) 'Hierba loza'.

(7074 FS.). Las Palmas, sección 3, muy escaso, en corte camino, sombrío. 17-10-78. Distr. predios: taludes sombríos. Distr. gral.: Bío-Bío a Cabo de Hornos.

\section{HIMENOPHYLLACEAE}

Himenophyllum dentatum. Cav. (H E herb)

Non col. Distr. predios: en bosque nativo secundario.

\section{DUECK y RODRIGUEZ, 1972;} VILLAGRAN ET AL., 1974.

Himenophyllum plicatum. Kaulf. (H E herb). Non col. Distr. predios: en bosque nativo secundario.

Serpyllopsis caespitosa. (Gand.) C. c.hr. (H E herb).

Non col. Distr. predios: en bosque nativo secundario.

\section{LOPHOSORIACEAE}

Lophosoria quadripinnata. (Gmel.) C.Chr. (H herb) 'Ampe'.

Non col. Distr. predios: orillas de cursos de agua, lugares húmedos y sombríos, sotobosque de bosque nativo secundario, abundante. Distr. gral.: Concepción a Magallanes.

\section{LYCOPODIACEAE}

Lycopodium paniculatum. Desv. ( $\mathrm{H}$ herb rept) 'Licopodio'.

(7075 FS.). Las Palmas, sección 3, muy escaso en taludes sombríos. 17-10-78. Arauco a Aysén.

LOOSER, 1962

POLYPODIACEAE

Polypodium feuillei. Bert. (H E herb) 'Calahuala'.

(7068 FS.). Los Pinos, sección 37, en corteza de E. cordifolia, abundante. 17-10-78. Distr. predios: epífita en árboles nativos, poco frecuente. Distr. gral.: Aconcagua a Aysén.

\section{GYMNOSPERMAE}

\section{PODOCARPACEAE}

Podocarpus salignus. D. Don (Meso P) 'Mañío de hojas largas'.

(313 OM.). Los Pinos, sección 23, Laguna Grande. 2-06-76. Distr. predios: ejemplares aislados en linderos de bosque nativo secundario, poco frecuente. Distr. gral.: Maule a Chiloé.

Saxegothaea conspicua Lindl. (Meso P) 'Mañío hembra'.

(7031 FS.). Los Pinos, sección 34, en la cima de la quebrada, muy escaso. 3-11-77. Distr. predios: fondo quebrada sección 34, muy escaso. Distr. gral.: Maule a Aysén.

\section{ANGIOSPERMAE}

\section{DICOTYLEDONEAE}

\section{AEXTOX1CACEAE}

Aextxicon punctatum. R. et Pav. (Meso P) 'Olivillo'.

(6786 FS.). Los Pinos, sección 31, Las Quemas, $P$. radiata 1971. 18-02-76. Distr. predios: bosque nativo secundario especialmente en quebradas, muy abundante. Distr. gral: Coquimbo a Chiloé.

\section{AMPELIDACEAE}

Cissus striata. R.et Pav. (el PL suff) 'Voqui parrilla'.

(339 A1.). Los Pinos, sección 37. 12-11-74. Distr. predios: trepadora en cercos y arbustos del matorral y en linderos de bosque nativo secundario, muy abundante. Distr. gral.: Valparaíso a Chiloé.

\section{APOCYNACEAE}

Elytropus chilensis. Muell. Arg. (st PL suff) 
'Quilmay'.

(280 Al.). Los Pinos, sección 34, P. radiata adulto. 3-01-74. Distr. predios: en bosque nativo secundario, poco frecuente. Distr. gral.: Maule a Chiloé.

\section{ARALIACEAE}

Pseudopanax laetevirens. (Gay) Franchet (Micro P) 'Sauco del diablo'.

Non col. Distr. predios: Las Palmas, sección 8 y 9, bosque nativo secundario, bosque pantanoso, escaso. Distr. gral.: Maule al Estrecho de Magallanes.

Pseudopanax valdiviensis. (Gay) Seemann (st P1 suff) 'Curaco'.

(121 OM.). Los Pinos, sección 23, Laguna Grande. 19-01-76. Distr. predios: trepadora facultativa en lugares sombríos, bosque nativo secundario, poco frecuente. Distr. gral.: Concepción a Chiloé.

\section{ASTERACEAE}

Baccharis elaeoides. Remy (NP) 'Chuca'.

(252 OM.). Los Pinos, sección 31, Las Quemas, $P$. radiata 1970. 11-03-76. Distr. predios: matorrales y plantaciones jóvenes, abundante. Distr. gral.: Arauco a Valdivia.

Baccharis racemosa. (DC.) (NP) 'Chilca'.

(163 OM.). Los Pinos, sección 25, P. radiata 1973. 27-01-76. Distr. predios: matorrales y plantaciones jóvenes, muy abundante. Distr. gral.: provincias centrales hasta Llanquihue.

Baccharis sagittalis. DC. (Ch suff) 'Verbena de 3 esquinas'.

(298 OM.). Las Palmas, sección 7, quebrada Patagüillas. 21-01-76. Distr. predios: quebradas y terrenos húmedos, poco frecuente. Distr. gral.: Coquimbo a Aysén.

Baccharis sphaerocephala. Hook. et Arn. (NP) 'Rari'.

(107 OM.). Los Pinos, sección 36, P. radiata 1975. 19-01-76. Distr. predios: matorrales y plantaciones, poco frecuente. Distr. gral.: Concepción a Chiloé.

Chevreulia sarmentosa. (Pers.) Blake (H).

(78 Al.). Los Pinos, sección 34, P. radiata 1971. 29-10-73. Distr. predios: plantaciones jóvenes y matorrales, poco frecuente. Distr. gral.: provincias centrales hasta Chiloé.

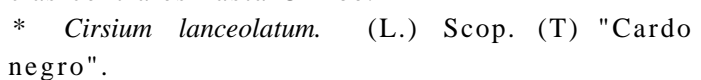

(6925 FS.). Los Pinos, sección 37, P. radiata 1976. 10-11-76. Distr. predios: matorrales, praderas y terrenos recién quemados, muy abundan- te.

Conyza. sp. (T).

(266 Al.). Los Pinos, sección 37, 3 - 1-74, abundante en matorrales.

* Crepis capillaris. (L.) Wallroth (T).

(6959 FS). Los Pinos, sección 31, Las Quemas, P. radiata 1970. 3-3-77. Distr. predios: matorrales, plantacionesjóvenes y praderas, muy abundante.

Gnaphalium cheiranthifolium. Lam. (T) "Té de burro".

(1390M.). Los Pinos, sección 36, P. radiata 1975. 27-01-76. Distr. predios: matorrales, plantaciones jóvenes y praderas, poco frecuente. Distr. predios: matorrales, plantaciones jóvenes y praderas, poco frecuente. Distr. gral.: Valparaíso a Valdivia.

Gnaphalium spiciforme. Sch. Bip. (T).

(164 OM.). Los Pinos, sección 25, P. radiata 1973. 27-01-76. Distr. predios: matorrales, plantaciones jóvenes y praderas, abundante. Distr. gral.: Valdivia a Magallanes.

* Hypochoeris radicata. L. (H ros) "Hierba del chancho".

(91 OM.). Los Pinos, sección 34, P. radiata 1971. 19-01-76. Distr. predios: matorrales, plantaciones jóvenes y praderas.

* Leontodon nudicaulis. (L.) Banks ex Lowe (H ros) "Chinilla".

(69 Al.). Los Pinos, sección 34, P. radiata 1971. 29-10-73. Distr. predios: matorrales y praderas, muy abundante.

CABRERA, 1949; MATTHEI, 1963.

Leptocarpha rivularis. DC. (NP) "Palo negro". (77 OM.). Los Pinos, sección 31, Las Quemas, P. radiata 1971. 19-01-76. Distr. predios: en matorrales, abundante. Distr. gral.: Maule a Llanquihue.

Mutisia retusa. Remy (el PL herb) "Clavel del campo".

(167 OM.). Los Pinos, sección 31, Las Quemas, P. radiata 1971. 19-01-76. Distr. predios: matorrales y plantaciones jóvenes, muy escaso. Distr. gral.: Concepción a río Palena.

Senecio cymosus. Remy (NP) "Palpalén".

(7067 FS.). Los Pinos, sección 36, P. radiata 1976, poco frecuente. 17-10-78. Distr. predios: matorral arbustivo de plantaciones jóvenes en sotobosque de $P$. radiata, poco frecuente. Distr. gral: Valdivia a Aysén.

Senecio otites. Kunze ex DC. (H scap) "Trompón".

* Especie introducida. 
(6780 FS ) Los Pinos, sección 31, Las Que mas, $P$ radiata 1971 8-02-76 Distr. predios matorrales y plantaciones jóvenes, muy abundante Distr. gral.: Arauco a río Palena.

* Senecio sylvaticus. L. (T) "Melosa'.

(6934 FS .). Los Pinos, sección 37 P. radiata 1976, poco frecuente. 10-11-76. Distr. predios: matorrales, plantacionesjovenes y praderas, abundante.

* Senecio vulgaris. L. (T).

(115 OM.). Los Pinos, sección 23, Laguna Grande. 19-01-73. Distr. predios: matorrales y praderas, abundante.

Senecio yegua. (Colla) Cabr. (NP) "Palo yegua".

(7310 FS ) Los Pinos, sección 29, P. radiata explotado 1979, poco frecuente. 28-10-79. Distr. predios: matorral arbustivo.

CABRERA, 1949.

* Siegesbeckia orientalis. L. (T) "Pega pega". (306 OM.). Las Palmas, sección 7, quebrada Patagüillas. 6-05-74. Distr. predios: lugares sombríos, linderos de bosque escaso.

* Sonchus asper (L.) Hill (T) "Ñilhue".

(254 OM.). Los Pinos, sección 31, Las Quemas, P. radiata 1971. 11-03-76. Distr. predios: matorrales en plantaciones jóvenes, orillas de caminos, muy abundante.

\section{BERBERIDACEAE}

Berberis buxifolia. Lam. (NP) "Calafate".

(311 A1.). Los Pinos, sección 23, Laguna Grande. 8-10-74. Distr. predios: matorrales poco densos, orillas de caminos, poco frecuente. Distr. gral.: Santiago a Tierra del Fuego.

Berberis darwinii. Hook. (NP) "Michay".

(15 Al.). Las Palmas, sección 6. 25-10-73.

Distr. predios: linderos de bosque nativo secundano y matorrales a orillas de caminos, poco frecuente. Distr. gral.: Linares a Aysén.

\section{BETULACEAE}

* Alnus glutinosa (L.) Gaertn. (Meso P) "Aliso negro".

(7091 FS.). Los Pinos, sección 22, orillas río Cayumapu, naturalizado, abundante 24-10-78.

\section{BIGNONIACEAE}

Campsidium valdivianum. (Phil.) Skottsb. (st PL suff) "Pilpil voqui".

(307 OM.). Las Palmas, sección 7, quebrada Patagüilla. 2-06-76. Distr. predios: trepadora voluble en árboles de bosques pantanoso, abundante. Distr. gral. Concepción a Magallanes.

\section{BORRAGINACEAE}

* Cynoglossum sp. (T sem)

(7027 FS.). Las Palmas, sección 6, Ps. menziesii 1973, poco frecuente. 20-10-77. Distr. predios: en matorrales abiertos, escaso.

\section{BUDDLEJACEAE}

Buddleja globosa. Lam. (NP) Matico"

(7085 FS.). Las Palmas, sección 7, quebrada Patagüillas, poco frecuente. 25-10-78. Distr. predios: en matorrales, poco frecuente. Distr. gral. Santiago a Chiloé.

\section{CALLITRICHACEAE}

* Callitriche autumnalis. L. (hyd T) "Estrella de agua".

(135 Al.). Las Palmas, sección 7, quebrada Patagüillas. 15-11-73. Distr. predios: en lugares de aguas lénticas, poco frecuente.

\section{CARYOPHYLLACEAE}

* Cerastium arvense. L. (Ch scap).

(6929 FS ). Los Pinos, sección 37, 14-11-76. Distr. predios terrenos quemados y matorrales abiertos, poco frecuente.

Stellaria cuspidata. Willd. (Ch scap) "Quilloiquilloi".

(79 OM.). Los Pinos, sección 31, Las Quemas, $P$. radiata 1970. 19-01-76. Distr. predios en terrenos quemados, abundante. Distr. gral.: Atacama a río Palena.

\section{CELASTRACEAE}

Maytenus boaria. Mol. (Meso P) "Maitén".

(305 A1.). Las Palmas, sección 6, bosque nativo secundario. 24-09-74. Distr. predios orillas de camino y linderos de bosque nativo secundario, poco frecuente. Distr. gral.: Coquimbo a Chiloé.

\section{CUNONIACEAE}

Caldcluvia paniculata. (Cav.) D. Don (Meso P) "Tiaca".

(7084 FS.). Las Palmas, sección 7, quebrada Patagüillas, poco frecuente 25-10-78. Distr. predios: bosques de galería, orillas de vertientes, escaso. Distr. gral.: Concepción a Chiloé.

Weinmannia trichosperma. Cav. (Meso P) "Tineo".

Non col. Distr. predios vegetación de quebradas, escaso. Distr. gral.: Maule a Chiloé.

\section{CONVOLVULACEAE}

* Dichondra sericea. SW. var servicea (H caesp) "Oreja de ratón".

(97 A1.). Las Palmas, sección 9, pradera. 7-11-73. Distr. predios: matorraly praderas, muy 
abundante.

\section{CRUCIFERAE}

* Brassica campestris L. (T) "Yuyo".

(106 Al.). Las Palmas, sección 7, quebrada Patagüillas. 7-11-73. Distr. predios praderas y superficies recientemente quemadas orillas de caminos, escasa.

* Cardamine nasturtioides. Bert. (T) "Berro". (136 A1.). Las Palmas, sección 7, Sequoia 1972. 15-11-73. Distr. predios: praderas, matorrales y plantaciones nuevas, poco frecuente.

* Raphanus sativus. L. (T) "Rábano silvestre". (7030 FS.). Los Pinos, sección 32, P. radiata 1977. 3-11-77. Distr. predios: plantaciones nuevas, orillas de caminos, escaso.

\section{ELAEOCARPACEAE}

Aristotelia chilensis. (Mol.) Stuntz (Micro P) "Maqui".

(324 A1.). Los Pinos sección 34, P. radiata 1971. 8-10-74. Distr. predios: matorrales y terre nos quemados, muy abundante Distr. gral.: Coquimbo a Chiloé.

\section{ERICACEAE}

Gaultheria phillyreifolia. (Pers.) Sleum. (NP) "Chaura no comestible".

(34 A1.). Las Palmas, sección 6, bosque nativo secundario. 25-01-73. Distr. predios matorrales y linderos de bosques, abundante. Distr. gral.: Curicó a Magallanes.

\section{ESCALLONIACEAE}

Escallonia revoluta (R. et P.)Pers. (NP)"Lun". (150 OM.) Las Palmas, sección 6, P. menziesii 1973. 27-01-76. Distr. predios: matorrales y terrenos húmedos, escaso. Distr. gral.: Coquimbo a Valdivia.

Escallonia rosea. Griseb. (NP) "Siete camisas".

(7034 FS.). Las Palmas, sección 7. 3-11-77. Distr. predios: matorrales a orillas de cursos de agua, escasa. Distr. gral.: Valdivia a Aysén.

\section{EUCRYPHIACEAE}

Eucryphia cordifolia. Cav. (Meso P) "Ulmo, Muermo".

(7069 FS.). Los Pinos, sección 37, poco frecuente 17-10-78. Distr. predios bosque nativo secundario, abundante. Distr. gral.: Arauco a Chiloé.

\section{FAGACEAE}

Nothofagus dombeyi. (Mirb.) Oerst. (Meso P) "Coigüe".

Non col. Distr. predios: bosque nativo secundario y vegetación de quebradas, muy abundante.
Distr. gral. Colchagua a Aysén.

Nothofagus obliqua. (Mirb.) Oerst. (Meso P) "Roble, Pellín".

(7072 FS.). Las Palmas, sección 9, pradera, abundante. 17-10-78. Distr. predios: bosque nativo secundario y vegetación de quebradas, muy abundante. Distr. gral. Aconcagua a Chiloé.

\section{FLACOURTIACEAE}

Azara integrifolia. R. et Pav. (NP) 'Chillán'

(22 A1.). Las Palmas sección 6, Ps. menziesii 1973. 25-10 73. Distr. predios: matorrales y orillas de caminos, escaso Distr. gral.: Aconcagua a Chiloé.

Azara lanceolata Hook. f. (NP) "Corcolen' (328 A1.). Los Pinos, sección 34, P. radiata 1971. 8-10-78. Distr. predios bosque nativo y matorrales en sombra, muy escaso. Distr. gral.: Arauco a Aysén.

\section{GERANIACEAE}

Geranium corecore. Steud. (H ros) "Corecore".

(106 OM.). Los Pinos, sección 25, P. radiata 1973. 19-01-76. Distr. predios. matorrales y praderas, abundante. Distr. gral.: provincias centrales hasta Valdivia.

Geranium patagonicum. Hook. f. (H).

(124 A1.). Las Palmas, sección 3, Abies alba 1973. 15-1 1-73. Distr. predios matorrales y plantaciones jóvenes, abundante . Distr. gral.: Valdivia al Estrecho de Magallanes.

\section{GESNERIACEAE}

Mitraria coccinea. Cav. (NP E suff) "Botellita".

(7048 FS.). Las Palmas, sección 7, quebrada Patagüillas, creciendo en el suelo 25-04-78. Distr. predios: en linderos de bosque y orillas de caminos en el suelo como trepadora, abundante. Distr. gral.: Maule al Estrecho de Magallanes.

Sarmienta repens. R. et Pav. (Ch E suff) "Medallita".

Non col. Distr. predios: epífita en bosque nativo, muy escasa. Distr. gral.: Maule a Chiloé, aisladamente en Fray Jorge.

\section{GUNNERACEAE}

Gunnera chilensis. Lam. (H ros) "Nalca".

Non col. Poco frecuente, en vertientes y lugares húmedos. Distr. gral.: Coquimbo a Magallanes.

\section{HYPERICACEAE}

Hipericum brevistylum. Choisy (H scap) "Ñanco".

(253 A1.). Los Pinos, sección 23, Laguna Grande 27-12-73. Distr. predios: en lugares húmedos, escaso. Distr. gral.: Concepción a Chiloé. 
* Hypericum humifusum, L. (T).

(7062 FS.). Los Pinos, sección 36, P. radiata 1976. 16-05-78. Distr. predios: estrato herbáceo de plantaciones jóvenes y praderas, abundante.

* Hypericum perforatum. L. (H) "Hierba de San Juan".

(130 Al.). Las Palmas, sección 3, 15-11-73. Distr. predios: estrato herbáceo de plantaciones jóvenes y praderas, poco frecuente.

\section{LABIATAE}

* Lycopus europaeus. L. (H) "Pata de lobo". (299 OM.). Los Pinos, sección 23, Laguna Grande. 9-04-76. Distr. predios: en lugares húmedos y sombríos, poco frecuente.

* Menta pulegium. L. (Ch scap) "Poleo".

(170 OM.). Los Pinos, sección 36, $P$. radiata 1975, 5-02-76. Distr. predios: matorrales y praderas, poco frecuente.

* Prunella vulgaris. L. (Ch) "Hierba mora".

(108 OM.). Los Pinos, sección 25, P. radiata 1973, orilla camino central. 19-01-76. Distr. predios: praderas y orillas de senderos, abundante.

Stachys bridgesii. Benth (Ch). "Hierba de Santa Rosa".

(132 OM.). Los Pinos, sección 23, Laguna Grande. 19-01-76. Distr. predios: matorrales plantaciones jóvenes, abundante. Distr. gral.: Concepción a Valdivia.

\section{LARDIZABALACEAE}

Boquila trifolioliata. DC. (st. PL suff) "Pilpil voqui".

(8 A1.). Las Palmas, sección 6, Ps. menziesii 1973. 25-10-73. Distr. predios: plantaciones jóvenes, matorrales y bordes de bosque nativo secundario, abundante. Distr. gral.: río Maule a Chiloé.

Lardizabala biternata. Done, (el PL suff) "Coile".

(290 A1.). Los Pinos, sección 34, P. radiata 1971. 7-06-74. Distr. predios: bosque nativo secundario y plantaciones adultas, muy escaso. Distr. gral.: Aconcagua a Chiloé.

\section{LAURACEAE}

Persea Lingue. Nees (Meso P) "Lingue".

Non col. Distr. predios: orillas de praderas y vegetación de quebradas. Distr. gral.: Cocalán a Palena

\section{LOASACEAE \\ Loasa acanthifolia. Desr. (H scap) "Ortiga brava".}

(7046 FS.). Las Palmas, sección 7, quebrada Patagüillas, C. lusitanica 1975. 25-04-78. Distr. predios: matorrales a linderos de bosque nativo secundario, abundante. Distr. gral.: Concepción a Chiloé.

\section{LOBELIACEAE}

Lobelia tupa. L. (H scap) "Tabaco del diablo", "Tupa".

(143 OM.). Los Pinos, sección 34, P. radiata 1971. 27-01-76. Distr. predios: matorrales y plantaciones jóvenes, abundante. Distr. gral.: provincias centrales y del sur del país.

\section{LORANTHACEAE}

Lepidoceras, kingii. Hook.f. (Ch suff parasit) "Quintral del temu".

(7045 FS.). Los Pinos, sección 23, Laguna Grande, en Blepharocalyx cruckshanksii poco frecuente. 19-03-78. Distr. predios: hemiparásito en temu de bosque pantanoso, abundante. Distr. gral.: Arauco a Chiloé.

Tristerix tetrandus. (R. et Pav.) Martius. (Ch suff parasit) "Quintral".

(304 Al.). Las Palmas, sección 6, bosque nativo secundario. 24-09-74. Distr. predios: matorrales, preferentemente en maqui, aveces en Acacia melanoxylon, poco frecuente. Distr. gral.: Coquimbo a Chiloé.

\section{MALVACEAE}

Corynabutilon ochsenii. Phil. (NP) "Huella chica".

(6773 FS.). Los Pinos, sección 31, Las Quemas, P. pinaster 1970, poco frecuente. 18-02-76. Distr. predios: en plantaciones jóvenes y matorrales, escaso. Distr. gral.: Valdivia a Osorno.

Corynabutilum vitifolium. (Cav.) Kearney (Micro P) "Huella".

(151 Al.). Los Pinos, sección 31, Las Quemas, $P$. radiata 1970 . 15-11-73. Distr. predios: matorrales y bordes de bosque. Distr. gral.: Arauco a Chiloé.
* Modiola caroliniana.
(L.) D. Don (H) "Pila pila".

(6938 FS.). Los Pinos, sección 37, P. radiata 1976, poco frecuente. 10-11-76. Distr. predios: estrato herbáceo de plantaciones jóvenes, poco frecuente.

\section{MIMOSACEAE}

* Acacia melanoxylon. R.Br. (Meso P) "Aromo australiano".

Non. col. Las Palmas y Los Pinos, naturalizado.

\section{MONIMIACEAE}

Laurelia philippiana Looser (Meso P) "Tepa", 
"Huahuán".

(7071 FS.). Las Palmas, sección 9, pradera, abundante. 17-10-78. Distr. predios: bosque nativo secundario, abundante. Distr. gral.: Valdivia a Aysén.

Laurelia sempervirens. (R. et Pav.) Tul. (Meso P) "Laurel".

(100 A1.). Las Palmas, sección 9, bosque nativo secundario. 7-11-73. Distr. predios: en bosque nativo secundario, abundante. Distr. gral.: Colchagua a Llanquihue.

\section{MYRTACEAE}

Amomyrtus luma. (Mol.) Legr. et Kaus. (Meso P) "Luma".

(127 OM.). Los Pinos, sección 23, Laguna Grande. 19-01-76. Distr. predios: bosque pantanoso y bosque nativo secundario, abundante. Distr. gral.: Maule a Aysén.

Amomyrtus meli. (Phil.) Legr. et Kaus. (Meso P) "Melí".

(7084 FS.). Las Palmas, sección 8, bosque nativo secundario, poco frecuente. 25-10-78. Distr. predios: bosque nativo secundario. Distr. gral: Cautín a Chiloé.

Luma apiculata. (DC.) Burret (Meso P) "Arrayán".

(311 OM.). Las Palmas, sección 7, quebrada Patagüillas. 2-06-76. Distr. predios: bosque pantanoso, muy abundante. Distr. gral.: Colchagua a Chiloé.

Luma gayana. (Barn.) Buriet. (Micro P) "Huillipeta".

(231 OM.). Las Palmas, sección 7, quebrada Patagüillas, bosque de galería. 3-03-76. Distr. predios: bosque de galería, muy abundante. Distr. gral.: Bío-Bío a Chiloé.

Myrceugenia exsucca. (DC.) Berg (Meso P) "Patagua".

(310 OM.). Las Palmas, sección 7, quebrada Patagüillas, bosque de galería. 2-06-76. Distr. predios: bosque pantanoso, muy abundante. Distr. gral.: Coquimbo a Chiloé.

Myrceugenia parvifolia. (DC.) Kaus. (NP) "Patagüilla".

(232 OM.). Las Palmas, sección 7, quebrada Patagüillas, bosque de galería. 3-03-76. Distr. predios: bosque pantanoso, muy abundante.

Blepharocalyx cruckshanksii. (H. et A.) Nied. (Meso P) "Temu".

(7049 FS.). Las Palmas, sección 7, quebrada Patagüillas. 25-04-78. Distr. predios: bosque pantanoso, muy abundante. Distr. gral.: Colchagua a Chiloé,

Ugni molinae. Turcz. (NP) "Murta".

(94 OM.). Los pinos, sección 34, P. radiata
1971. 1901-76. Distr. predios: matorrales y orillas de caminos, muy abundante. Distr. gral.: Talca a río Palena.

KAUSEL, 1942 .

\section{OLEACEAE}

* Fraxinus ornus. L. (Micro P) "Fresno de flor". (7031 FS.). Los Pinos, sección 32, naturalizado. Distr. predios: Los Pinos, sección 25 y 34, $P$. radiata, poco frecuente.

\section{ONOGRACEAE}

Fuchsia magellanica. Lam. var magellanica (NP) "Chuco".

(90 OM.). Los Pinos, sección 39, P. radiata 1971. 19-01-76. Distr. predios: lugares húmedos, muy abundante. Distr. gral.: Coquimbo a Tierra del Fuego.

\section{OXALIDACEAE}

Oxalis aureoflava Steud. (H rept) "Vinagrillo".

(6874 FS.). Las Palmas, sección 7, quebrada Pataguillas, abundante. 13-12-76. Distr. predios: estrato herbáceo de plantaciones jóvenes, muy abundante. Distr. gral.: Centro Sur de Chile.

Oxalis dumetorum. Barn. (H) "Culle".

(224 A1.). Las Palmas, sección 7, quebrada Patagüillas. 13-12-73. Distr. predios: en lugares húmedos de bosque nativo secundario, poco frecuente. Distr. gral.: Valdivia a Chiloé.

Oxalis rosea. Jacq. (T) "Culle colorado".

(158 OM.). Los Pinos, sección 23, Laguna Grande. 27-01-76. Distr. predios: en lugares sombríos, poco frecuente. Distr. gral.: Coquimbo a Chiloé.

Oxalis valdiviensis. Barn. (T).

(6875 FS.). Las Palmas, sección 7, quebrada Patagüillas. C. lusitanica 1975, poco frecuente. 13-05-76. Distr. predios: orillas de cursos de agua y lugares con sombra, poco frecuente. Distr. gral.: provincia de Valdivia.

\section{PAPILIONACEAE}

* Cytisus scoparius. L. (NP) "Lluvia de oro". (18 Al.). Las Palmas, sección 6, Ps. menziesii 1973, naturalizado. 25-10-73. Distr. predios: matorrales de plantaciones jóvenes, poco frecuente. Distr. gral.: Constitución a Coyhaique.

Lotus subpinnatus. (Lag.) (H) "Porotillo".

(174 Al.). Los Pinos, sección 23, Laguna Grande. 15-11-73. Distr. predios: en praderas, poco frecuente. Distr. gral.: Antofagasta a Valdivia. * Lotus uliginosus. Schkuhr (H rept) "Alfalfa chilota".

(110 OM.). Los Pinos, sección 36, P. radiata 1975. 19-01-76. Distr. predios: praderas y mato- 
rrales, abundante.

* Teline monspessulana. (L) K. Koch (NP) "Retamillo".

(153 OM.). Los Pinos, sección 31, Las Quemas, $P$. radiata 1971 , muy abundante, naturalizado. 27-01-76. Distr. predios: matorrales y sotobosque de $P$. radiata, orillas de caminos, muy abundante. Distr. gral.: Aconcagua a Valdivia.

* Trifolium dubium. Sibth. (T).

(6928 FS.). Los Pinos, sección 37, P. radiata 1976, poco frecuente. 10-11-76. Distr. predios: praderas y matorral de plantaciones nuevas, poco frecuente.

* Trifolium pratense. L. (H scap) "Trébol rosado".

(89 OM.). Los Pinos, sección 34, P. radiata 1971. 19-01-76. Distr. predios: praderas y matorrales, poco frecuente.

* Trifolium repens. L. (H caesp) "Trébol blanco".

(149 OM.). Las Palmas, sección 6, Ps. menziesii 1973. 27-01-76. Distr. predios: en praderas y matorrales, poco frecuente.

* Sophora microphylla. Ait. (Micro P) "Pelú".

(7070 FS.). Las Palmas, sección 9, poco frecuente. 17-10-78. Distr. predios: bordes de bosque nativo secundario y cerca de orillas de cursos de agua, escaso. Distr. gral.: Maule a Aysén.

* Ulex europaeus. L. (NP) "Aliaga", "Espino", "Yaquil".

(17 Al.). Las Palmas, sección 5, Ps. menziesii naturalizado. 25-01-73. Distr. predios: matorrales y plantaciones jóvenes, poco frecuente. Distr. gral: Concepción a Chiloé.

* Vicia angustifolia. L. (T) "Arvejilla".

(6908 FS.). Los Pinos, sección 36, P. radiata 1976, poco frecuente. 26-10-76. Distr. predios: matorrales y terrenos quemados, poco frecuente.

* Vicia atropurpurea. Desf. (T) "Arvejilla".

(119 Al.). Las Palmas, sección 3, Abies alba 1973. 15-11-73. Distr. predios: praderas y matorrales, poco frecuente.

* Vicia cf. hirsuta. S.F. Gray (T) "Arvejilla". (6937 FS.). Los Pinos, sección 37, P. radiata 1976, poco frecuente. Distr. predios: praderas y matorrales, poco frecuente.

\section{PHYTOLACCACEAE}

Ercilla volubilis. A. et H.L. Juss. (d PL suff) "Voqui-auca".

(7033 FS.). Los Pinos, sección 23, Laguna Grande, muy escaso. 3-11-77. Distr. predios: bajo bosque nativo secundario abierto, en ambientes húmedos, muy escaso. Distr. gral.: Concepción a Chiloé.

\section{PLANTAGINACEAE}

* Plantago lanceolata. L. (H ros) "Siete venas". (146 OM.). Los Pinos, sección 36, Ps. menziesii 1971. 27-01-76. Distr. predios: praderas y plantaciones jóvenes, muy abundante.

\section{POLYGONACEAE}

* Rumex acetosella. L. (H ros) "Acetosa".

(136 OM.). Los Pinos, sección 25, P. radiata 1973. 27-01-76. Distr. predios: terrenos quemados, praderas y plantaciones, muy abundante.

* Muehlenbeckia hastulata. (J.E.Sm.) Johnst. (d PL suff) "Quilo", "Mollaca".

(263 Al.). Los Pinos, sección 34, $P$. radiata 1971. 3-01-74. Distr. predios: trepadora en matorrales, plantaciones jóvenes y linderos, abundante. Distr. gral.: Coquimbo a Chiloé.

\section{PORTULACACEAE}

Calandrinia axilliflora. Barn. (T succ).

(6932 FS.). Los Pinos, sección 37, P. radiata 1976. 10-11-76. Distr. predios: solamente en terrenos recién quemados, abundante. Distr. gral.: Aconcagua a Valdivia.

\section{PROTEACEAE}

Embothrium coccineum. J.R. et G. Forts. (Meso P) "Notro".

(109 Al.). Las Palmas, sección 7, quebrada Pataguillas, bosque de galería. 7-11-73. Distr. Predios: quebradas, matorrales, plantaciones jóvenes y linderos, abundante. Distr. gral.: Maule a Tierra del Fuego.

Gevuina avellana. Mol. (Meso P) "Avellano".

Non col. Distr. predios: bosque nativo secundario, abundante. Distr. gral.: Valparaíso a Chiloé.

Lomatia dentata. (R. et Pav.) R. Br. (Micro P) "Piñol", "Avellanillo".

(308 OM.). Las Palmas, sección 7, quebrada Patagüillas, bosque de galería. 2-06-76. Distr. predios: matorrales, orillas de caminos y linderos, poco frecuente. Distr. gral.: Aconcagua a Palena.

Lomatia ferruginea. (Cav.) R. Br. (Micro P) "Fuinque".

(312 OM.). Las Palmas, sección 7, quebrada Pataguiillas, bosque de galería. 2-06-76. Distr. predios: bosque de galería, escaso. Distr. gral.: Curicó a Magallanes.

Lomatia hirsuta. (Lam.) Diels (Meso P)

"Radal".

Non col. Distr. predios: matorrales, plantaciones jóvenes y linderos, abundante. Distr. gral. Lomatia hirsuta. (Lam.) Diels (Meso P) "Radal".

Non col. Distr. predios: matorrales, planta- 
ciones jóvenes y linderos, abundante. Distr. gral.: Coquimbo a Chiloé.

\section{RANUNCULACEAE}

Ranunculus minutiflorus. Bert. (H rept)

(118 A1.). Las Palmas, sección 3, Abies alba 1973. 15-11-73. Distr. predios: praderas y plantaciones jóvenes, abundante. Distr. gral: provincias centrales hasta Tierra del Fuego.

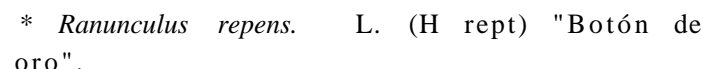

(165 OM.). Las Palmas, sección 7, quebrada Patagüillas. 27-01-76. Distr. predios: vegas y praderas húmedas, muy abundante.

\section{RHAMNACEAE}

Rhamnus diffusus. Clos. (NP) "Murta negra". (122 OM.). Los Pinos, sección 23, Laguna Grande. 19-01-76. Distr. predios: bosque pantanoso y en sotobosque de Pinus radiata, en bosque nativo secundario, poco frecuente.

\section{ROSACEAE}

Acaena ovalifolia. R. et Pav. (H rept) "Cadi$110 "$.

(81 OM.). Los Pinos, sección 31, Las Quemas, P. radiata 1970. 19-01-76. Distr. predios: matorrales, plantaciones jóvenes, linderos y praderas, muy abundante. Distr. gral.: Coquimbo a Tierra del Fuego.

Fragaria chiloensis. (L.) Ehrh. (H rept) "Frutilla".

(74 A1.). Los Pinos, sección 34, P. radiata 1971. 29-10-73. Distr. predios: praderas, poco frecuente. Distr. gral.: Concepción a Aysén.

* Rosa eglanteria. L. (NP) "Mosqueto".

(348 A1.). Los Pinos, sección 34, P. radiata 1971. 12-11-74. Distr. predios: praderas, poco frecuente. Distr. gral.: Valparaíso a Aysén.

* Rubus constrictus. Lef. et Mey. (d PL suff) "Murra".

(168 A1.). Los Pinos, sección 31, Las Quemas, $P$. radiata 1970 , naturalizado. Distr. predios: praderas, linderos y sotobosque de $P$. radiata, muy abundante. Distr. gral.: Arauco a Aysén.

\section{RUBIACEAE}

Leptostigma arnotianum. Valp. (G riz).

(7047 FS.). Las Palmas, sección 7, quebrada Patagüillas. 25-04-78. Distr. predios: praderas y orillas de senderos. Distr. gral.: Valdivia a Osorno.

Nertera granadensis. (Mutis ex L.f.) Drude (H rept) "Coralito".

(302 A1.). Las Palmas, sección 6, bosque nativo secundario. 24-9-74. Distr. predios: bosque nativo secundario, lugares húmedos y taludes de caminos, muy abundante. Distr. gral.: Maule a Tierra del Fuego.

Relbunium hypocarpium. (L.) Hemsl.

(Ch) "Relbún".

(154 OM.). Los Pinos, sección 31, Las Quemas, P. radiata 1970. 27-01-76. Distr. predios: matorrales, plantaciones jóvenes y orillas de caminos, muy abundante. Distr. gral.: Aconcagua a Chiloé.

\section{SALICACEAE}

* Salix caprea. L. (Meso P) "Sauce gatito".

(100 OM.). Los Pinos, sección 36, Ps. menziesii 1971, naturalizado. 19-01-76. Distr. predios: plantaciones jóvenes y lugares húmedos, poco frecuente. Distr. gral.: Valparaíso a Coyhaique.

\section{SANTALACEAE}

Myoschilos oblonga. R. et Pav. (NP) "Codocoipu".

(82 OM.). Los Pinos, sección 31, Las Quemas, $P$. radiata 1970. 19-01-76. Distr. predios: bordes de senderos, matorrales, plantaciones jóvenes y linderos de bosques, escaso. Distr. gral.: Aconcagua a Magallanes.

\section{SAXIFRAGACEAE}

Ribes punctatum. R. et Pav. (NP) "Parrilla".

(288 OM.). Los Pinos, sección 36, 7-06-74. Distr. predios: matorrales, plantaciones jóvenes y linderos, poco frecuente. Distr. gral.: Aconcagua hasta Chiloé.

\section{SCROPHULARIACEAE}

* Bellardia trixago. (L.) All. (T).

(207 A1.). Las Palmas, sección 9, pradera. 13-12-73. Distr. predios: praderas y plantaciones jóvenes, poco frecuente.

* Digitalis purpurea. L. (T) "Dedalera".

(260 A1.). Los Pinos, sección 34, P. radiata 1971. 3-01-74. Distr. predios: matorrales, plantaciones jóvenes y linderos, abundante.

Mimulus luteus. L. (H caesp) "Placa".

(252 A1.). Los Pinos, sección 23 Laguna Grande. 27-12-73. Distr. predios: orillas de cursos de agua, como planta palustre, muy escasa. Distr. gral.: Coquimbo a Magallanes.

* Veronica serpillifolia L. (H caesp) "Verónica".

(113 Al.). Las Palmas, sección 7, quebrada Patagüillas, bosque de galería. 7-12-73. Distr. predios: praderas y plantaciones jóvenes, abundante.

\section{SOLANACEAE}

Solanum gayanum. Remy (NP) "Natri". (6911 FS.). Los Pinos, sección 36, P. radiata 
1976. 26-10-76. Distr. predios: borde de caminos, matorrales, y plantaciones jóvenes, escasa. Distr. gral.: Valdivia a Chiloé.

* Solanum nigrum. L. (T) "Tomatillo".

(7079). Los Pinos, sección 34, abundante. 19-10-78. Distr. predios: orillas de caminos y terrenos recién quemados, poco frecuente.

Solanum valdiviense. Dun. (NP) "Llagecillo". (80 OM.). Los Pinos, sección 31, Las Quemas, P. radiata 1976. 19-01-76. Distr. predios: bordes de caminos, matorrales y plantaciones jóvenes, muy abundante. Distr. gral.: Valdivia a Chiloé.

\section{TROPAEOLACEAE}

Tropaeolum speciosum. Poepp. et. Endl. (st GL) "Pajarito".

(6779 FS.). Los Pinos, sección 31, Las Quemas, Ps. menziesii 1970, muy escaso. 18-02-76. Distr. predios: matorrales, plantaciones jóvenes y linderos, muy escaso. Distr. gral.: Concepción a Chiloé.

\section{UMBELLIFERAE}

* Centella asiatica. (L.) Urb. (H rept).

(7092 FS.). Los Pinos, sección37, abundante . 24-10-78. Distr. predios: terrenos de bosque explotado y quemado y praderas húmedas, muy abundante.

Hydrocotyle hirta. $\mathrm{R} . \mathrm{Br}$. (h rept).

(7061 FS.). Los Pinos, sección 36, P. radiata 1976. 16-05-78. Distr. predios: matorrales y praderas, abundante. Distr. gral.: Concepción a Chiloé.

Hydrocotyle poeppigii. DC. var poeppigii (H rept) "Tembladerilla".

(70 OM.). Los Pinos, sección 31, Las Quemas, P. radiata 1970. 19-01-76. Distr. predios: matorrales y praderas, muy abundante. Distr. gral.: Arauco a Llanquihue.

Osmorhiza chilensis. Hook et Arn. (H scap) "Asta de cabra".

(213 Al.). Las Palmas, sección 7, bosque nativo secundario. 13-12-73. Distr. predios: bajo bosque nativo secundario, muy abundante. Distr. gral.: Coquimbo a Tierra del Fuego.

\section{URTICACEAE}

Pilea elegans. Rich. (H scap).

(300 OM.). Los Pinos, sección 23, Laguna Grande. 9-04-76. Distr. predios: orillas decursos de agua y lugares muy húmedos y sombríos, abundante. Distr. gral.: Valdivia a Osorno.

Pilea ellyptica. Hook.f. (H scap).

(304 OM.). Las Palmas, sección 7, quebrada Patagüillas. 6-05-76. Distr. predios: orillas de cursos de agua y lugares muy húmedos y sombríos, abundante. Distr. gral.: Valdivia a Chiloé.

\section{VERBENACEAE}

Rhaphithamnus spinosus. (A. Juss) Moldenke (Micro P) "Arrayán macho".

(35 A1.). Las Palmas, sección 6, bosque nativo secundario. 25-10-73. Distr. predios: bosque nativo secundario y matorrales, abundante. Distr. gral.: Coquimbo a Aysén.

\section{VIOLACEA E}

Viola maculata. Cav. (H ros) "Pilludén".

(44 A1.). Los Pinos, sección 31, Las Quemas, P. radiata 1970. 29-10-73. Distr. predios: matorrales, plantaciones jóvenes y linderos, abundante. Distr. gral.. Río Rapel al Estrecho de Magallanes.

Viola rubella. Cav. (Ch) "Violeta del monte".

(38 A1.). Las Palmas, sección 6, bosque nativo secundario. 25-10-73. Distr. predios: superficies sombrías, orillas de caminos y linderos. Distr. gral.: Concepción a Chiloé.

* Viola tricolor. L. (T) "Trinitaria".

(6936 FS.). Los Pinos, sección 37, P. radiata 1976. 10-11-76. Distr. predios: praderas y plantaciones jóvenes, muy escaso.

\section{WINTERACEAE}

Drimys winteri. Forst. (Meso P) "Canelo".

(307 A1.). Los Pinos, sección 23, Laguna Grande. 8-10-74. Distr. predios: bosque de galería y quebradas, muy abundante. Distr. gral.: Coquimbo a Cabo de Hornos.

\section{MONOCOTYLEDONEAE}

\section{ALSTROEMERIACEAE}

Alstroemeria aurantiaca. D.Don (a G rad) "Amancai".

(92 OM.). Los Pinos, sección 34, $P$. radiata 1971. 19-01-76. Distr. predios: matorrales, plantaciones jóvenes y orillas de caminos, abundante. Distr. gral.: Talca a Chiloé.

\section{BROMELIACEAE}

Fascicularia bicolor. (R et Pav.) Mez. (H E) "Chupalla".

Non col. Distr. predios: bosque nativo secundario, epífita, escasa. Distr. gral.: Valdivia a Chiloé (Islas Guaitecas).

Greigia sphacelata. R. et Pav.) Regel (H herb) "Chupón".

Non col. Distr. predios: bosque nativo secundario, matorrales y plantaciones de $P$. radiata, abundante. Distr. gral.: Concepción a Llanquihue. 


\section{CYPERACEAE}

Carex fuscula. D'Urv. (H).

(369 OM.). Los Pinos, sección 31, Las Quemas, $P$. radiata 1970 . 13-01-77. Distr. predios: terrenos pantanosos, muy abundante. Distr. gral.: Valparaíso a Chiloé.

Cyperus eragrostis. Lam. (e $\mathrm{H}$ caesp) "Cortadera".

(118 OM.). Los Pinos, sección 23, Laguna Grande. 19-01-76. Distr. predios: bosque pantanoso, terrenos húmedos, muy abundante. Distr. gral.: Valparaíso a Llanquihue.

Eleocharis pachycarpa. Desv. (e $\mathrm{H}$ caesp).

(67 A1.). Los Pinos, sección 23, Laguna Grande. 29-10-73. Distr. predios: bosque pantanoso, terrenos húmedos, muy abundante. Distr. gral.: provincias del sur del país.

Scirpus inundatus. (R. Br.) Poir. (e H caesp) "Can-can".

(125 OM.). Los Pinos, sección 23, Laguna Grande. 19-01-76. Distr. predios: bosque pantanoso, terrenos húmedos, muy abundante. Distr. gral.: provincias del sur del país.

Uncinia erinacia. (Cav.) Pers. (e $\mathrm{H}$ caesp) "Quinquín".

(104 A1.). Las Palmas, sección 9, bosque nativo secundario. 7-11-73. Distr. predios: matorrales, plantaciones jóvenes y bosque nativo secundario, abundante. Distr, gral.: Valdivia y Osorno.

Uncinia phleoides. (Cav.) Pers. (e H caesp) "Quinquín".

(87 OM.). Los Pinos, sección 34, P. radiata 1971. 19-01-76, Distr. predios: matorrales, plantaciones jóvenes y bosque nativo secundario, muy abundante, Distr, gral,: Valparaíso a Chiloé.

\section{DIOSCOREACEAE}

Dioscorea helicifolia. Kunth, (st GL) "Papa cimarrona".

(370 OM.), Los Pinos, sección 31, Las Quemas, $P$. radiata 1970. 13-01-77. Distr. predios: matorrales y plantaciones, escaso. Distr. gral,: Coquimbo a Chiloé.

\section{GRAMINEAS}

* Agrostis castellana. Boiss, et Reut. (e H rept) "Chépica".

(166 OM.). Las Palmas, sección 7, quebrada Patagüillas. 27-01-76. Distr. predios: praderas y plantaciones jóvenes, muy abundante.

* Agrostis tenuis. Sibth. (e H rept) "Pasto qui$1 \mathrm{a}^{\prime}$.

(273 Al.). Los Pinos, sección 31, Las Quemas, $P$. radiata 1970. 3-01-74. Distr. predios: praderas y plantaciones jóvenes, abundante.
* Aira caryophyllea. L. (T).

(6788 FS.). Los Pinos, sección 31. 18-02-76.

Distr. predios: matorrales y prados.

* Anthoxanthon odoratum. L. (e $\mathrm{H}$ caesp) "Pasto oloroso".

(99 OM.). Los Pinos, sección 36, Ps. menziesii 1971. 19-01-76. Distr. predios: praderas y plantaciones jóvenes, muy abundante.

* Arrhenatherum elatius. var. bulbosum (Willd.) Spenner (e H caesp).

(320 OM.). Los Pinos, sección 36, Ps. menziesii 1971. 8-10-76. Distr. predios: matorrales, plantaciones jóvenes y praderas, poco frecuente.

* Briza minor. L. (T) "Tembladera".

(188 A1.). Las Palmas, sección 6, Ps. menziesii 1973. 13-12-73. Distr. predios: plantaciones jóvenes, matorrales y praderas, poco frecuente.

Briza stricta. (Hook. et Arn.) Steud. (e H caesp). (7064 FS.). Los Pinos, sección 36, P. radiata 1976. 16-05-78. Distr. predios: praderas y plantaciones jóvenes. Distr. gral.: Valparaíso a Valdivia.

Bromus catharticus. Vahl (H) "Lanco".

(204 Al.). Las Palmas, sección 6, Ps. menziesii 1973. 13-12-73. Distr. predios: praderas y plantaciones jóvenes, poco frecuente. Distr. gral.: Coquimbo a Llanquihue.

Chascolytrun subaristatum. (Lam.) A.N. Desv. (T).

(135 OM.). Los Pinos, sección 36, radiata 1976. 27-01-1976. Distr. predios: plantaciones jóvenes, matorrales abiertos y praderas, poco frecuente, Distr. gral.: Bío-Bío a Valdivia.

Chasquea quila. (Mol.) Kunth. (Micro P) o (d PL frut) "Quila".

(6691 FS.). Las Palmas, sección 9, bosque nativo secundario, 20-06-75. Distr, predios: arbusto trepador en bosque nativo secundario, plantaciones adultas, matorrales y linderos, muy abundante. Distr. gral,: Cautín a Chiloé (Isla Desertores).

* Cynosorus echinatus. L. (T) "Cebadilla".

(112 OM.). Los Pinos, sección 23, Laguna Grande, 19-01-76. Distr, predios: linderos, plantaciones jóvenes y praderas, poco frecuente.

* Dactylis glomerata. L. (e $\mathrm{H}$ caesp) "Pasto ovillo".

(152 OM.), Las Palmas, sección 6, Ps. menziesii 1973. 27-01-76. Distr. predios: praderas y plantaciones jóvenes, abundante.

* Danthonia sp. (T) "Cepilla".

(6964 FS.). Los Pinos, sección 36, P. radiata 1976. 3-05-77. Distr. predios: matorrales y plantaciones jóvenes, abundante.

Elymus gayanum. Desv. (H).

(101 OM.). Los Pinos, sección 25, P. radiata 
1973. 19-01-76. Distr. predios: matorrales y plantaciones jóvenes, escaso. Distr. gral.: Santiago a Aysén.

* Festuca rubra. L. (e H caesp) "Festuca".

(140 A1.). Los Pinos, sección 34, P. radiata 1971. 15-11-73. Distr. predios: praderas, matorrales y plantaciones jóvenes, poco frecuente.

* Holcus lanatus. L. (e H caesp) "Pasto miel". (198 A1.). Las Palmas, sección 6. 13-12-73. Distr. predios: matorrales, praderas y plantaciones jóvenes, muy abundante.

* Lolium perenne. L. (H) "Ballica inglesa".

(6907 FS.). Los Pinos, sección 36, P. radiata 1976. 20-10-76. Distr. predios: matorrales, plantaciones jóvenes y praderas, poco frecuente.

Nasella chilensis. (Trin. et. Ripr.) Desv. (e H caesp).

(157 OM.). Las Palmas, sección 6, Ps. menziesii 1970. 21-01-76. Distr. predios: matorrales y plantaciones jóvenes, abundante. Distr. gral.: Colchagua a Valdivia.

Nasella juncea. Phil. (e $\mathrm{H}$ caesp).

(58 A1.). Los Pinos, sección 31, Las Quemas, P. radiata 1970. 23-10-73. Distr. predios: matorrales y plantaciones jóvenes, poco frecuente.

Piptochaetium montevidense. (Spreg.) Parodi (e H caesp) "Coironcillo".

(244 Al.). Los Pinos, sección 31, Las Quemas, $P$. radiata 1970. 27-12-73. Distr. predios: matorrales, plantaciones jóvenes y bordes de caminos, poco frecuente. Distr. gral.: provincicias del centro y sur del país.

* Poa annua. L. (T) "Pasto piojillo".

(93 Al.). Las Palmas, sección 9, pradera. 7-11-73. Distr. predios: praderas y plantaciones jóvenes, abundante.

* Poa pratensis. L. (e H caesp) "Pasto azu1".

(6938 FS.). Los Pinos, sección 36, P. radiata 1976. 28-11-76. Distr. predios: praderas, matorrales y plantaciones jóvenes, poco frecuente.

* Polypogon australis. Brong. (H) "Cola de zorro".

(230 OM.). Los Pinos, sección 23, Laguna Grande. 3-03-76. Distr. predios: terrenos húmedos, abundante.

Relchela panicoides. Steud. (T).

(111 OM.). Los Pinos, sección 23, Laguna Grande. 19-01-76. Distr. predios: matorrales y praderas, abundante. Distr. gral.: Valparaíso a Llanquihue.

Stipa poeppigiana. Trin. et Rupr. (e H caesp) "Quilmén".

(6961 FS.). Los Pinos, sección 36, P. radiata 1976. 3-03-77. Distr. predios: matorrales y plantaciones jóvenes, abundante. Distr. gral.: Cautín a Llanquihue.

\section{IRIDACEAE}

Libertia chilensis, (Mol.) GUNCKEL (e H caesp) "Calle-calle".

(72 OM.). Los Pinos, sección 31, Las Quemas, P. radiata 1970. 19-01-76. Distr. predios: bosque nativo secundario, matorrales, plantaciones jóvenes y linderos, cerca de lugares húmedos, muy abundante. Distr. gral.: Maule a Chiloé.

Libertia sessiliflora. (Poepp.) Skott sb. (e H caesp).

(7028 FS.). Los Pinos, sección 31, Las Quemas, $P$. radiata 1970. Distr. predios: matorrales, plantaciones jóvenes y linderos, escaso. Distr. gral.: Aconcagua a Chiloé. 3-11-77.

Sisyrinchium graminifolium. Lindl. (e G rad) "Huilmo".

(194 Al.). Los Pinos, sección 23, Laguna Grande. 23-11-73. Distr. predios: praderas y matorrales, poco frecuente. Distr. gral.: Antofagasta a Magallanes.

Sisyrinchium cf. iridifolium. H.B.K. e G rad.

(6960 FS.). Los Pinos, sección 36, P. radiata 1976. Distr. predios: terrenos explotados, poco frecuente. Distr. gral.: Maule a Palena.

JUNCACEAE

* Juncus bufonius. L. (T).

(123 Al.). Las Palmas, sección 3, Abies alba 1973. 15-11-73. Distr. predios: superficies húmedas, muy abundante. Distr. gral.: Curicó a Aysén.

Juncus microcephalus. H.B.K. (H).

(123 OM.). Los Pinos, sección 31, Laguna Grande. 19-01-76. Distr. predios: bosque pantanoso y lugares húmedos, muy abundante. Distr. gral.: Maule a Llanquihue.

Juncus planifolius. $\mathrm{R} . \mathrm{Br}$. (T).

(6965 FS.). Los Pinos, sección 36, P. radiata 1976. 3-03-77. Distr. predios: bosque pantanoso y lugares húmedos, abundante. Distr. gral.: Santiago a Llanquihue.

Juncus procerus. C.E. Mey (e H caesp) "Junquillo".

(133 OM.). Los Pinos, sección 23, Laguna Grande, abundante. Distr. gral.: Maule a Magallanes.

Juncus stipularis. Nees et meyen ( $\mathrm{T}$ ).

(123 OM.). Los Pinos, sección 23, Laguna Grande. 27-01-76. Distr. predios: bosque pantanoso y lugares húmedos, abundante. Distr. gral.: Curicó a Magallanes.

ORCHIDACEAE

Codonorchis lessonii. Lindl. (ear G rad) "Azucena".

(32 A1.). Las Palmas, sección 6, bosque nativo secundario. 25-10-73. Distr. predios: bosque na- 
tivo secundario, linderos y matorrales, poco frecuente. Distr. gral.: Chillán a Tierra del Fuego.

Gavilea araucana. (Phil.) Correa (ear G rad).

(7043 FS.). Los Pinos, sección 32, bajo Fraxinus ornus, abundante solamente en ese lugar. 15-12-77. Distr. predios: sotobosque a linderos, muy escaso. Distr. gral.: Curicó a Valdivia.

Spiranthes diuretica. Lindl. (ear G rad).

(7044 FS.). Las Palmas, sección 3, P. radiata 1968. 22-03-78. Distr. predios: matorrales, praderas y linderos, muy escaso. Distr. gral.: O'Higgins a Aysén.

\section{PHILESIACEAE}

Lapageria rosea. R. et Pav. (st PL suff) "Copihue".

(7065 FS.). Los Pinos, sección 24. 17-10-78. Distr. predios: bosque nativo secundario, linderos y plantaciones, abundante. Distr. gral.: Valparaíso a seno de Reloncaví.

Luzuriaga polyphylla. (Hook.) Machr. "Quilineja".

(7080 FS.). Las Palmas, sección 7, quebrada Patagüillas. 25-10-78. Distr. predios: epífita en bosque pantanoso, bosque nativo secundario, poco frecuente. Distr. gral.: Valdivia a Chiloé.

Luzuriaga radicans. R. et Pav. (PL) "Quilineja".

(129 OM.). Los Pinos, sección 23, Laguna Grande. 19-01-76. Distr. predios: bosque de galería, bosque nativo secundario, epífita muy abundante. Distr. gral.: Chillán a Chiloé.

URBAN, 1934; BUENDIA, 1965; MUÑOZ, 1966; ZANDER, 1972; NAVAS, 1973; MARTICORENA Y QUEZADA, 1985.

\section{RESULTADOS Y DISCUSION}

La flora de los alrededores de Cayumapu se compone de 213 especies vasculares, de las cuales 150 son nativas $(70.4 \%)$ y 63 son taxones introducidos y naturalizados $(29.6 \%)$. En el grupo de las nativas se destacan 30 especies arbóreas, de ellas 21 alcanzan gran tamaño (más de 15 m.) y solamente dos de ellas son coniferas. Se encontraron además cuatro especies arbóreas introducidas, naturalizadas. Las especies arbustivas sumaron 27 y de ellas cuatro corresponden a arbustos introducidos naturalizados.

Comparando el espectro biológico de la flora estudiado con aquel del Parque Nacional V. Pérez Rosales (Villagrán et al., 1974), llama la atención que este último, con un total de 180 taxones, sólo comprende cuatro introducidos $(2,2 \%)$ mientras que en Cayumapu asciende a un 29,6\%. Las especies introducidas que participan en la flo- ra de Cayumapu corresponden preponderantemente a los grupos de las Terófitas y Hemicriptófitas con 12,2 y $11,3 \%$ respectivamente (Cuadro 1).

Las especies herbáceas anuales introducidas constituyen el 72,2 \% del total de las Terófitas. Esta situación demostraría la prolongada perturbación antrópica del área de Cayumapu, donde el desplazamiento de la vegetación original ha.permitido la invasión de especies introducidas, especialmente anuales. Esto concuerda con Schmithüsen, (1956) y Schlegel (1966), quienes explican en forma similar situaciones aún más extremas para el matorral esclerófilo de la Zona Central de Chile.

En la flora nativa de Cayumapu lideran los fanerófitos con 53 especies (35,3\%) junto a los Hemicriptófitos con 51 especies (34\%).

Al comparar el espectro biológico de Cayumapu con aquel del matorral esclerófilo de Maipú, Zona Central de Chile (Schlegel 1966), las diferencias climáticas entre ambos lugares de estudio quedan reflejadas en una destacada diversidad florística de especies arbóreas (30 contra 7 especies) y una representación muy moderada de Geófitas y Terófitas nativas (19 contra 123 especies) para la flora de Cayumapu.

Las especies arbustivas introducidas y naturalizadas Teline monspesulana, Rubus constrictus, Ulex europaeus, Cytisus scoparius y Rosa eglanteria, son extraordinariamente abundantes en los terrenos cubiertos por matorrales y plantaciones recientes. Las dos primeras además proliferan conspicuamente en el sotobosque de plantaciones de Pinus radiata y Eucalyptus globulus de mayor edad (Ramírez et al., 1984). La fuerte tendencia de esas especies arbustivas hacia una proliferación aún mayor, hace necesario el estudio del manejo de la vegetación, tendiente a su limitación.

El estudio florístico deja en evidencia que una serie de especies nativas son extraordinariamente escasas en el lugar, reflejando con ello un alto grado de intervención.

Las más destacadas entre ellas son: Licopodium paniculatum, Gleichenia quadripartita, Saxegothaea conspicua, Tropaeolum speciosum, Ercilla volubilis, Gavilea araucana, Spiranthes diuretica, Solanum gayanum y Corynabutilon ochsenii. Algunas de ellas nunca han sido muy abundantes en la región.

Finalmente, se puede concluir que la riqueza florística constatada hace del área estudiada un lugar de alto interés para investigaciones taxonómica y ecológicas. 
Cuadro 1, Espectro biológico de los predios Los Pinos y Las Palmas, Cayumapu, Valdivia

Tabla 1, Biological Spectrum of the Forest Farms Los Pinos and Las Palmas, Cayumapu, Valdivia.

\begin{tabular}{|c|c|c|c|c|c|}
\hline Forma de vida & $\begin{array}{l}\mathrm{N}^{\circ} \text { de especies } \\
\text { nativas }\end{array}$ & $\%(+)$ & $\begin{array}{l}\mathrm{N}^{\circ} \text { especies } \\
\text { introducidas }\end{array}$ & $\% \quad(++)$ & Total \\
\hline Mesofanerófito & 21 & 14,0 & 3 & 1,4 & 24 \\
\hline M icrofanerófito & 9 & 6,0 & 1 & 0,5 & 10 \\
\hline Nanofanerófito & 23 & 15,3 & 4 & 1,9 & 27 \\
\hline Caméfito & 8 & 5,3 & 3 & 1,4 & 11 \\
\hline Hemicriptófito & 51 & 34,0 & 24 & 11,3 & 75 \\
\hline Lianas o trepadoras & 11 & 7,3 & 2 & 0,9 & 13 \\
\hline Epífitas & 8 & 5,3 & - & - & 8 \\
\hline Geófitas & 9 & 6.0 & - & - & 9 \\
\hline Terófitas & 10 & 6,8 & 26 & 12,2 & 36 \\
\hline TOTAL & 150 & 100.00 & 63 & 29,6 & 213 \\
\hline
\end{tabular}

(+) Porcentaje relativo al total de especies nativas.

(++) Porcentaje referido al total de especies.

\section{SIGNIFICADO DE LAS ABREVIATURAS}

a) Formas de vida.

\begin{tabular}{|c|c|c|c|}
\hline Meso P & esofanerófitos: árboles grandes, & Ch E & Caméfitos epífitos. \\
\hline Micro P & $\begin{array}{l}\text { 5-50 m. } \\
\text { Microfanerófitos: árboles peque- }\end{array}$ & Ch suff parasit & $\begin{array}{l}\text { Caméfitos subfrutescentes hemi- } \\
\text { parásitos. }\end{array}$ \\
\hline NP & $\begin{array}{l}\text { ños, } 2-5 \mathrm{~m} \text {. } \\
\text { Nanofanerófitos: arbustos meno- }\end{array}$ & Ch E suff & $\begin{array}{l}\text { Caméfitos subfrutescentes epífi- } \\
\text { tos. }\end{array}$ \\
\hline $\mathrm{Ch}$ & $\begin{array}{l}\text { res a } 2 \mathrm{~m} \text {. } \\
\text { Caméfitos: plantas leñosas cuyos }\end{array}$ & Ch E herb & $\begin{array}{l}\text { Caméfitos subfrutescentes herbá- } \\
\text { ceos. }\end{array}$ \\
\hline & $\begin{array}{l}\text { sistemas de vástagos se mantienen } \\
\text { perenne hasta } 50 \mathrm{~cm} \text {., o plantas } \\
\text { mayores a } 50 \mathrm{~cm} \text {., pero cuyos ta- } \\
\text { llos periódicamente se secan has- } \\
\text { ta esa altura. }\end{array}$ & $\begin{array}{l}\text { Ch herb rept } \\
\mathrm{H}\end{array}$ & $\begin{array}{l}\text { Caméfitos herbáceos rastreros. } \\
\text { Plantas perennes herbáceas con } \\
\text { una reducción periódica de los } \\
\text { vástagos hasta la superficie del } \\
\text { suelo en el período de receso; }\end{array}$ \\
\hline Ch caesp & $\begin{array}{l}\text { Caméfitos subfrutescentes: arbus- } \\
\text { tos enanos semileñosos, leñosos } \\
\text { en la base del sistema de vastagos. } \\
\text { Caméfitos cespitosos. }\end{array}$ & H scap & $\begin{array}{l}\text { con sistema remanente de tallos } \\
\text { en la superficie del suelo. } \\
\text { Hemicriptófitos escaposos sin ro- } \\
\text { seta basal. }\end{array}$ \\
\hline Ch herb & $\begin{array}{l}\text { Caméfitos herbáceos: incluye a } \\
\text { todas las hierbas perennes, siem- } \\
\text { preverdes no leñosas, pastos y } \\
\text { helechos menores a } 1 \text { m. o siem- } \\
\text { preverdes por lo menos en } 25 \mathrm{~cm} \text {. } \\
\text { durante el período de receso. }\end{array}$ & $\begin{array}{l}\mathrm{H} \text { ros } \\
\mathrm{H} \text { rept } \\
\mathrm{H} \text { caesp } \\
\text { eH caesp }\end{array}$ & $\begin{array}{l}\text { Hemicriptófitos con roseta basal. } \\
\text { Hemicriptófitos rastreros. } \\
\text { Hemicriptófitos cespitosos. } \\
\text { Hemicriptófitos parcialmente } \\
\text { siempreverdes en el período de } \\
\text { receso, cespitosos. }\end{array}$ \\
\hline
\end{tabular}




eH rept
PL
el PL her
el PL suff
st PL suff
d PL suff
d PL frut

PE

G

a G rad Geófitos de raíces perennes, ver-
des en verano.

ear G rad Geófitos de raíces perennes, verdes en primavera.

st G L Geófitos trepadores.

$\mathrm{T}$ Terófitos: plantas anuales, cuyos sistemas de vástagos y raíces mueren después de la producción de semillas.

T sem Terófitos escaposos, semirosetados con hojas en el tallo.

T scap Terófitos escaposos sin roseta.

$\mathrm{T}$ su cc Terófitos suculentos.

hydr T Hidrófitos natantes.

\section{b) Colectores}

$\begin{array}{ll}\text { Al } & \text { Alumnos (E. Muñoz y V. Valdés). } \\ \text { FS } & \text { Federico Schlegel. } \\ \text { OM } & \text { Osvaldo Martínez. } \\ \text { Non col. } & \text { No ha sido colectado, pero su } \\ & \text { Eresencia ha sido comprobada. } \\ & \text { Especie introducida. }\end{array}$

introd.

\section{AGRADECIMIENTOS}

Por la asistencia en la comprobación de las identificaciones se agradece al Dr. O. Matthei y C. Marticorena, Instituto de Botánica de la Universidad de Concepción, y al Dr. C. Ramírez y H. Klempau, Instituto de Botánica de la Universidad
Austral de Chile.

Este estudio fue posible gracias al apoyo financiero del Centro Experimental Forestal (CEFOR) de la Facultad de Ciencias Forestales de la Universidad Austral de Chile.

\section{REFERENCIAS}

BUENDIA, F. 1965. Introducción al estudio de las especies pascícolas españolas. Inst. Forest. Invest. y Exp. Madrid.

CABRERA, A. L. 1949. "El género Senecio en Chile". Lilloa 15: 27-501.

DUEK, J. J. y RODRIGUEZ, R. 1972. "Lista preliminar de las especies de Pteridófitas en Chile continental e insular". Bol. Soc. Biol. Concepción XLV: 129-175.

FUENZALIDA, H. 1965. "Clima". En Geografía Económica de Chile: 99-151. Texto refundido. Corfo, Santiago, Chile.

HAUMANN. L. 1913. "La foret valdivienne et ses limites". Rec. Inst. Bor. IX, Bruselas.

HUBER, A. 1970. Diez años de observaciones climatológicas en la estación Teja-Valdivia (Chile) 1 960-69. Universidad Austral de Chile. Fac. de Ciencias. 46 pp.

KAUSEL, E. 1942. "Contribución al estudio de las mirtáceas chilenas". Rev. Arg. Agronomía 9 (3): 221243.

IREN. 1978. Estudios de los suelos de la prov. de Valdivia. Universidad Austral de Chile, Santiago de Chile. $178 \mathrm{pp}$.

LOOSER, G. 1962. "Los Pteridófitos o helechos de Chile". Rev. Univ. XLVII: 17-21. U. Católica, Santiago, Chile.

MARTICORENA, C. y QUEZADA, M. 1985. "Catálogo de la flora vascular de Chile". Gayana 42 (1-2) 157 MATTHEI, O. 1963. Manual ilustrado de las malezas de la provincia de $\tilde{N} u b l e$. Universidad de Concepción. $116 \mathrm{pp}$.

MONTALDO, P. 1966. Estudios ecológicos básicos de la provincia de Valdivia. I. Las Formaciones vegetales. Fac. C. Agr., Universidad Austral de Chile. Bol. Téc. $\mathrm{N}^{\circ} 2$.

MUELLER-DOMBOIS, D.; ELLEMBERG, H. 1974. Aims and methods of vegetation ecology. Ed. Wiley, London. 547 pp.

MUÑOZ, C. 1966. Sinopsis de la flora chilena. 2 ed. Ediciones U. de Chile, Santiago.

NAVAS, E. 1973. Flora de la cuenca de Santiago de Chile. Ed. de la Universidad de Chile, Santiago.

OBERDORFER. E. 1960. Pflanzensoziologische Studiem in Chile. Ed. J. Cramer, Karlsruke.

PARODI, L. R. 1947. "Las especies de gramíneas del género Nassella de la Argentina y Chile". Darviniana 7 (3): 369-395.

RAMIREZ, C. FERRIERE, F. y FIGUEROA, H. 1983. "Estudio Fitosociológico de los bosques pantanosos templados del sur de Chile". Rev. Chil. Hist, Nat. 56:11-26. RAMIREZ, C; FIGUEROA, H; CARRILLO, R. y CONTRERAS, D. 1984. "Estudio Fitosociológico de los estratos inferiores en un bosque de Pino". Bosque 5 (2): 65-81. 
REICHE, K. 1934. Geografía Botánica. Trad. G. Looser. Vol. I. 423 pp.

SCHLEGEL, F. 1966. "Pflanzensoziologische und floristische Untersuchungen über Hartlaubghoele im La Plata-Talbei. Santiago". Ber. Oberh. Ges. Nat. 34(3-4): 183-204.

URBAN, O. 1934. Botánica de las plantas endémicas de Chile. Impr. Concepción.

Recibido: 12-06-1987.
VILLAGRAN, G.; SEREY, L. y SOTO, C. 1974. "Catálogo de las plantas vasculares colectadas en el Parque Nacional "Vicente Pérez Rosales" ". Ann. Mus. Hist. Nat. 75-124. Valparaíso.

ZANDER. 1972. Handwoerterbuch der Pflanzennamen. Ed. Ulmer, Stuttgart. 